\title{
Tarife im Kreuzfeuer der Gesundheitspolitik
}

\section{Patrick Müllera, Christian Oeschger ${ }^{b}$, Thomas Kessler}

${ }^{a} \mathrm{FMH}$, Abteilungsleiter, Abteilung Ambulante Versorgung und Tarife; ${ }^{\mathrm{b}} \mathrm{FMH}$, Projektleiter, Abteilung Ambulante Versorgung und Tarife;

${ }^{\circ} \mathrm{FMH}$, Experte, Abteilung Ambulante Versorgung und Tarife

Am 28. September 2017 fand in Bern unter dem Titel «Tarife im Kreuzfeuer der Gesundheitspolitik» der zweite Tarifdelegierten-Tag dieses Jahres statt. An diesem Tarifdelegierten-Tag nahmen mehr als 100 Teilnehmer teil. Ein neuer Teilnehmerrekord!

Dr. med. Urs Stoffel begrüsste die Anwesenden und eröffnete den Tarifdelegierten-Tag mit einer standespolitischen Standortbestimmung und dem Überblick über die Ziele, die bevorstehende Thematik der Referate und der Podiumsdiskussion.

Stoffel sprach einleitend über die Kostenentwicklung im Gesundheitswesen und deren Ursache und erläuterte, warum die ambulanten Tarife gerade jetzt im Kreuzfeuer der Gesundheitspolitik stehen.

Zuletzt berichtete der Departementsverantwortliche für die «ambulante Versorgung und Tarife» über den gegenwärtigen Stand des Projekts TARCO. Das Projekt läuft nun seit über 365 Tagen. Mit der Annahme des Grobkonzeptes in der Delegiertenversammlung vom September 2016 erfolgte der Startschuss von TARCO. Seit der Delegiertenversammlung vom Januar 2017 konnten bereits einige Meilensteine von TARCO erreicht werden. Die Überarbeitung der Nomenklatur ist fast vollständig abgeschlossen und die Verhandlungen über die Kostenmodelle mit den Tarifpartnern sind gestartet.

Alle Unterlagen sowie die Gesamtpräsentation finden Sie auf der Website der FMH: www.fmh.ch $\rightarrow$ Ambulante Tarife $\rightarrow$ Tarifdelegierten-Tag $\rightarrow$ 28. September $2017 \rightarrow$ Präsentation

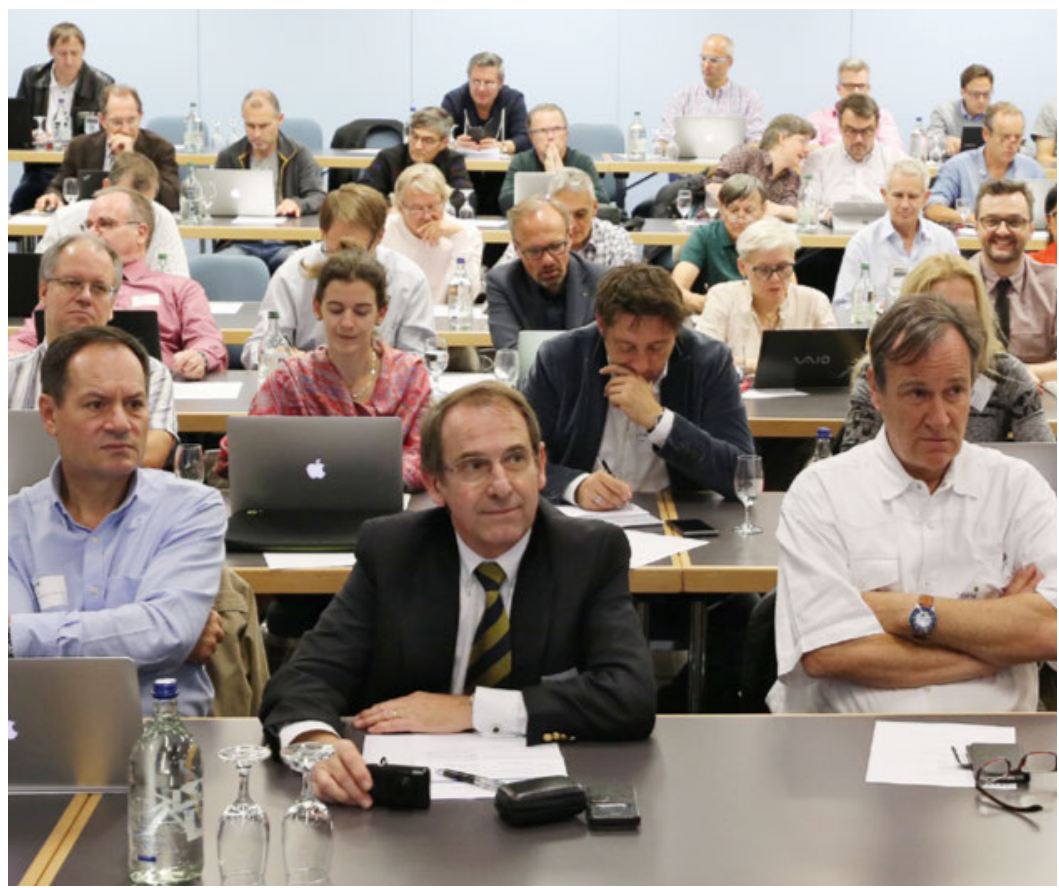

Der Tarifdelegierten-Tag fand in den Räumlichkeiten des Hotels Ador in Bern statt.

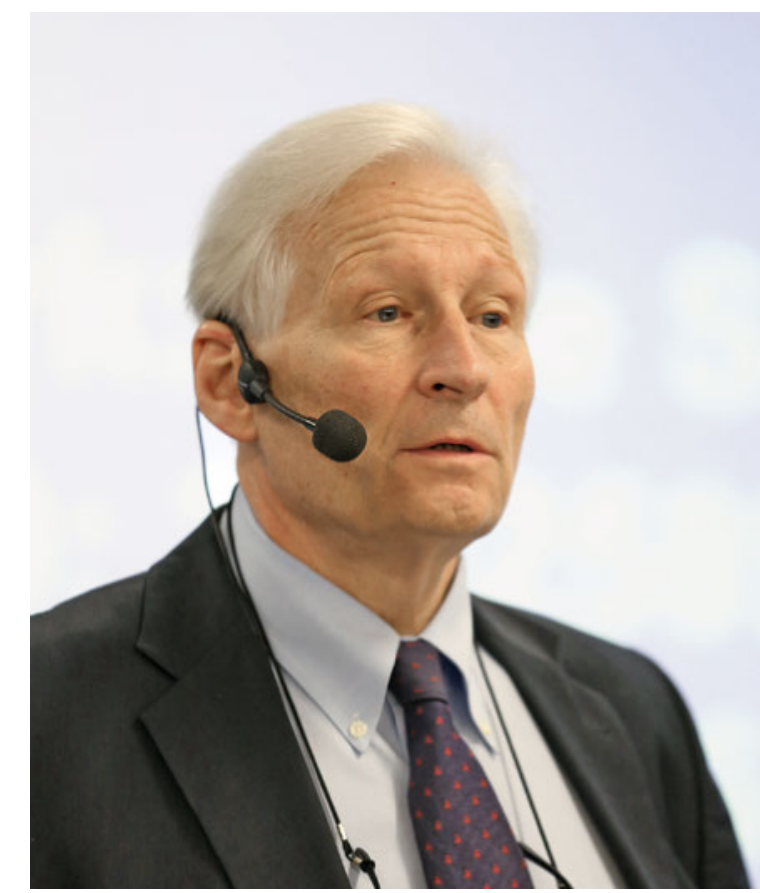

Dr. med. Urs Stoffel begrüsst die Teilnehmer des Tarifdelegierten-Tages. 


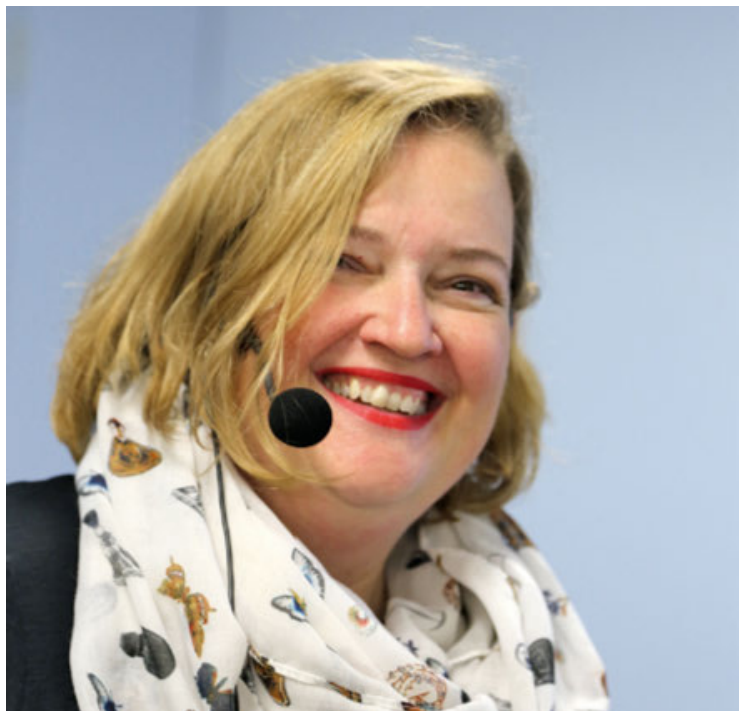

Dr. med. Susanne Christen referiert über die aktuellen Gegebenheiten im Bereich Praxislabor.

\section{Aktuelles im Bereich Praxislabor}

Dr. med. Susanne Christen informierte über den Bereich Praxislabor. Aktuell wird im Projekt transAL die Analysenliste nach 2009 erneut revidiert. Das Projekt sieht vor, obsolete und mehrfach tarifierte Positionen aus dem Tarif zu eliminieren.

Zudem informierte Christen über die im Parlament vorliegende Motion Hess (Geschäftsnummer 16.3193). Die Motion sieht vor, Artikel 52 des Bundesgesetzes über die Krankenversicherung (KVG) so zu ändern, dass die Tarife von Analysen durch medizinische Labors künftig - analog TARMED und DRG - durch die Tarifpartner verhandelt oder bei Unstimmigkeiten, durch eine von ihnen eingesetzte Rekurs-Instanz festgelegt werden. Weiter fordert die Motion Kuprecht (16.3487) die Einführung der Vertragsfreiheit bei den Labortarifen.

Ausserdem berichtete Christen über die aktuellen Gegebenheiten in der Schweizerischen Kommission für Qualitätssicherung in medizinischen Laboren (QUALAB).

\section{Zweiter Tarifeingriff des Bundesrates in den TARMED}

Patrick Müller informierte über den zweiten Tarifeingriff des Bundesrates in die Tarifstruktur TARMED und machte aufgrund immer noch fehlender Informationen seitens der Bundesbehörden einen Rückblick auf die Entwicklungen der letzten Jahre und die damit verbundene subsidiäre Kompetenz des Bundesrates. Die jahrelange Blockade und das Nichtzustandekommen einer Revision waren die Hintergründe dieser neuen erweiterten Kompetenz des Bundesrates. Der Bundesrat kann seit Anfang
2013 aktiv in eine bestehende inkraftgesetzte Tarifstruktur eingreifen - dies mittels einer Verordnung. Die Kompetenz beschränkt sich auf den ambulanten Bereich des Krankenversicherungsgesetzes (KVG).

Der Bundesrat fällte am 16. August 2017 einen Grundsatzentscheid. Dabei hält der Bundesrat am Tarifeingriff per 1. Januar 2018 fest. Der Bundesrat hat aber Korrekturen gegenüber der Vernehmlassung vorgenommen - dies auch aufgrund der über 600 eingegangenen Stellungnahmen, die vorwiegend aus Kreisen der Ärztinnen und Ärzte und der betroffenen Fachgesellschaften stammten.

Für Müller ist klar, dass mit dem Tarifeingriff die eigene Bundesratsstrategie «Gesundheit 2020» sowie Bundesprogramme wie beispielsweise Strategie Palliative Care, NCD-Strategie, Nationale Demenzstrategie, Strategie Sucht, damit vom Bundesrat torpediert werden. Der Tarifeingriff führt insgesamt zu einer deutlichen Schwächung der ambulanten Medizin, setzt keine Anreize für die Erbringung der Leistungen im deutlich günstigeren und effizienteren ambulanten Bereich und ist weder sachgerecht noch betriebswirtschaftlich.

\section{Projekt TARCO - aktueller Stand und Informationen}

Sabine Zehnder und Christian Oeschger informierten über den aktuellen Stand des Projektes TARCO. Das Cockpit, als oberstes Steuerungs- und Entscheidungsorgan des Projekts, setzt sich zusammen aus Vertretern aller Dachverbände der FMH sowie Vertretern der Kantone und des VLSS. Das Cockpit hat bis heute mehr als 60 Entscheide in vier Sitzungen gefällt und das Projekt somit vorwärts gebracht und entscheidend geprägt.

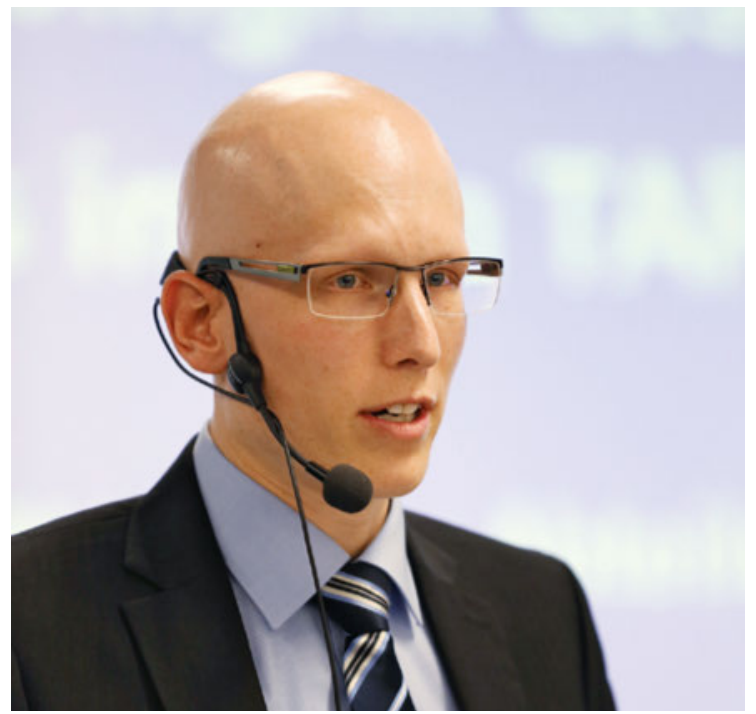

Patrick Müller informiert über den zweiten Tarifeingriff des Bundesrates in den TARMED. 

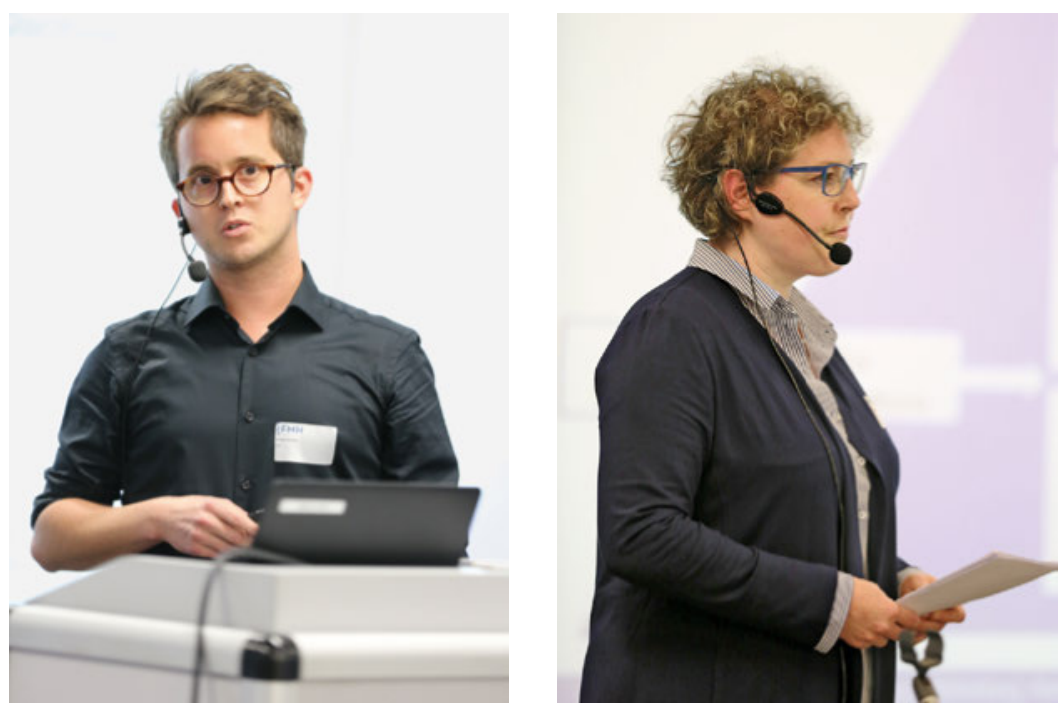

Christian Oeschger und Sabine Zehnder berichten über die aktuellen Arbeiten im Rahmen des Projektes TARCO.

Die Arbeitsgruppen haben aktuell noch bis Ende Oktober 2017 Zeit, die Nomenklatur zu finalisieren. Danach wird die gesamte Nomenklatur durch das Cockpit abgesegnet und der Expertengruppe der FMH ein Mandat zur weiteren Verhandlung mit den Tarifpartnern erteilt.

Zehnder informierte über die ärztlichen und nichtärztlichen Grundleistungen, die Behandlungs- und Beratungsleistungen, die Plausibilisierung der Handlungsleistungen und über die Arbeitsgruppen «Dignitäten» und «OP».

\section{Tarifpartner unterwegs zu einer gemeinsamen Tarifstruktur: Wunschgedanken oder Realität?}

Zur Podiumsdiskussion unter der Leitung von Prof. Urs Brügger vom Winterthurer Institut für Gesundheitsökonomie hat das Departement eine hochkarätige Runde eingeladen: Pius Zängerle (Direktor curafutura), Verena Nold (Direktorin santésuisse), Dr. med. Urs Stoffel (FMH-Zentralvorstand), Josef Müller (Vorstandsmitglied H+), Dr. med. Josef Brandenberg (Präsident $\mathrm{fmCh}$ ) und Dr. med. Philippe Luchsinger (Präsident mfe) diskutierten über Wunschgedanken und Realität im Zusammenhang mit der Revision des ambulanten Ärztetarifes.

Prof. Brügger fragte die Podiumsteilnehmer zum Einstieg, was für eine Vision sie zum ambulanten Tarif hätten. Brügger wollte von der Runde wissen, wie am Tag nach der Verabschiedung und Einreichung einer gemeinsamen und partnerschaftlichen Tarifstruktur der Titel der Zeitungen lauten könnte. Es stellt sich heraus, dass alle Podiumsteilnehmer sich mehr oder wenig einig sind, dass die Weiterentwicklung der Tarifstruktur von grosser Bedeutung ist und es wichtig ist, dass die fünf Tarifpartner einen Kompromiss finden. Der Titel der Zeitungen könnte folgendermassen lauten: «Die Tarifpartner haben sich beim Tarif TARMED geeinigt.» Für die Podiumsteilnehmer ist es zudem wichtig, dass es eine gemeinsame Organisation der Tarifpartner gibt, die den Tarif kontinuierlich weiterentwickelt

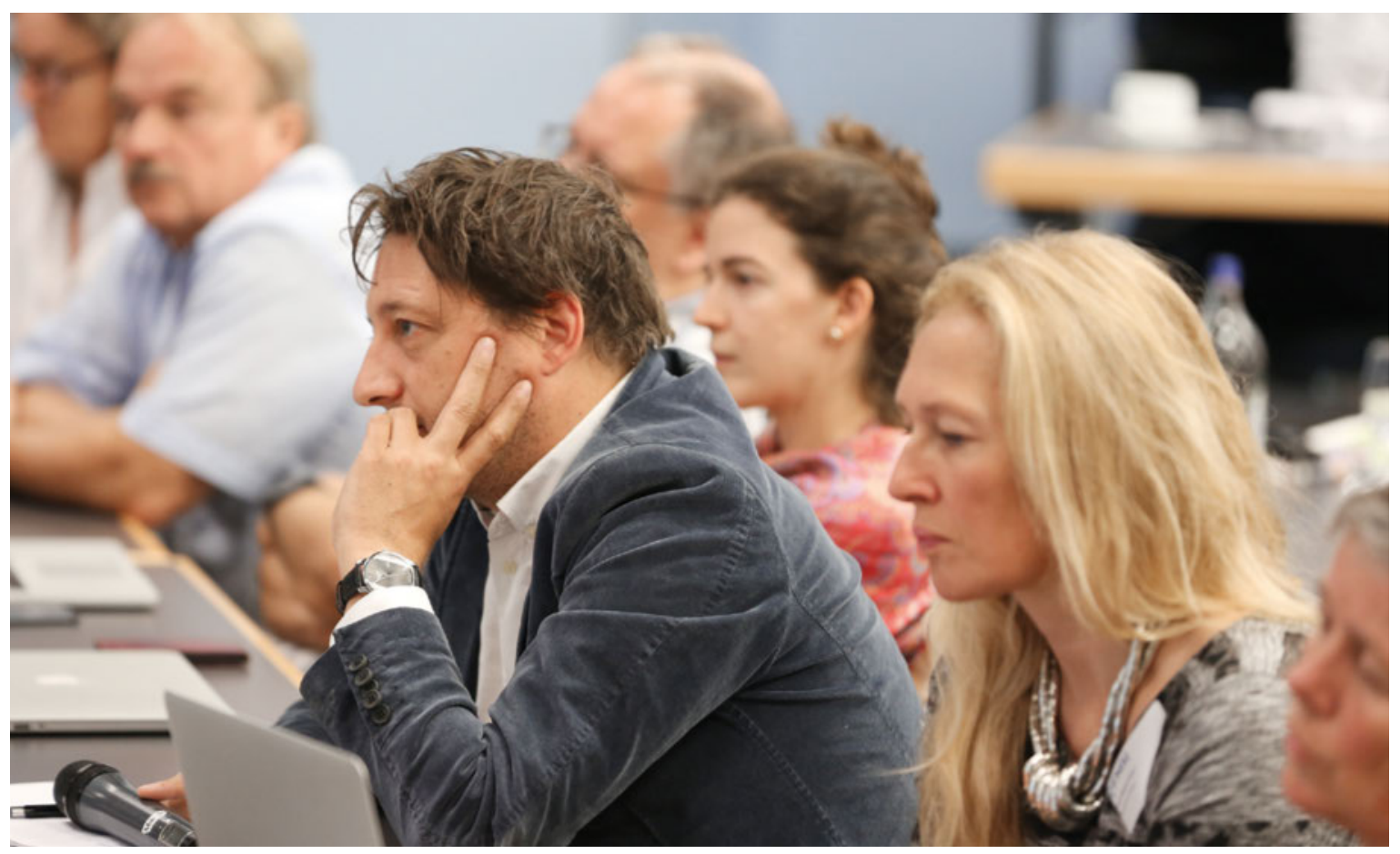

Die Referate stossen bei den Teilnehmern des Tarifdelegierten-Tages auf grosses Interesse. 


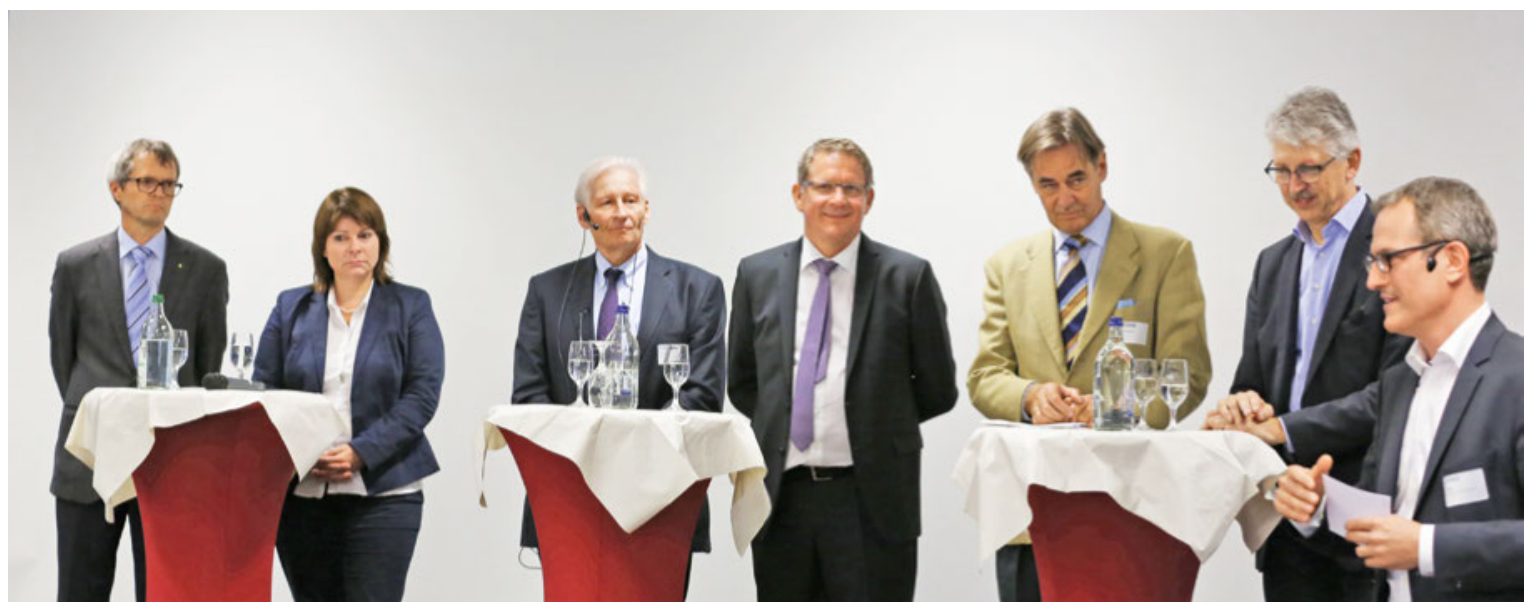

Prof. Dr. Brügger (rechts) moderierte die Podiumsdiskussion.

und pflegt, analog der SwissDRG AG. Unterschiedliche Auffassungen gibt es bezüglich der Art der Tarifierung der Leistungen - die einen wollen einen umfassenden Einzelleistungstarif und die anderen wollen vermehrt Pauschalen einsetzen, sodass der Tarif weniger Tarifpositionen aufweist. Für alle Podiumsteilnehmer ist letztlich klar, dass alle Tarifpartner Abstriche und Kompromisse machen müssen, sodass das Ziel «ein neuer Tarif» partnerschaftlich erreicht werden kann.

\section{Rückblick und Fazit}

FMH, Abteilung Ambulante Versorgung und Tarife Baslerstrasse 47 $\mathrm{CH}-4600$ Olten Tel. 0313591230 Fax 0313591238 tarife.ambulant[at]fmh.ch
Dr. Urs Stoffel zog zum Schluss der Veranstaltung Bilanz und formulierte folgendes Fazit: Der Druck seitens Politik wird weiter zunehmen. Die Bevölkerung wird durch die steigenden Prämien in der Wahrnehmung noch sensibler und ungeduldiger. Das Projekt TARCO ist wohl eine der letzten Chancen für eine gemeinsame und partnerschaftliche Gesamtrevision des TARMED. Stoffel ist überzeugt, dass die Ärzteschaft diese Chance und Möglichkeit unbedingt nutzen muss und nur so beweisen kann, dass sie willens ist, weiterhin aktiv an einer Tarifpartnerschaft festzuhalten. Das bedingt aber, dass alle Seiten bereit sind, Kompromisse einzugehen. «Kämpfen wir also gemeinsam und geschlossen für einen gemeinsamen Verhandlungstarif anstelle eines staatlich verordneten Amtstarifs».

\section{Bildnachweis}

Fotos Andreas Weissenburger

Der nächsteTarifdelegierten-Tag findet am Donnerstag, 26. April 2018 im Hotel Bern in Bern statt. Bitte reservieren Sie sich den Termin! 